05

\title{
Анализ условий устойчивости плоского скопления дислокаций, заторможенного упругим полем клиновой дисклинации
}

\author{
(C) В.В. Рыбин, ${ }^{1,2}$ В.Н. Перевезенцев, ${ }^{1}$ Ю.В. Свирина ${ }^{1, \uparrow}$ \\ ${ }^{1}$ Институт проблем машиностроения РАН, \\ 603024 Нижний Новгород, Россия \\ ${ }^{2}$ Санкт-Петербургский политехнический университет Петра Великого, \\ 195251 Санкт-Петербург, Россия \\ e-mail: j.svirina@mail.ru \\ (Поступило в Редакцию 10 июля 2017 г.)
}

Проведен анализ равновесной конфигурации плоского скопления краевых дислокаций в суммарном поле внешних и внутренних, создаваемых клиновой стыковой дисклинацией, упругих напряжений. Рассмотрены условия потери устойчивости скопления и прохождения пластического сдвига через силовой барьер, создаваемый дисклинацией.

DOI: 10.21883/JTF.2018.07.46167.2420

\section{Введение}

Пластическая деформация поликристаллов сопровождается появлением на изломах и стыках границ зерен линейных дефектов ротационного типа - стыковых дисклинаций деформационного происхождения (СД ДП) [1,2]. Влияние СД ДП на процессы структурообразования и разрушения ранее рассматривали в работах $[3,4]$. Было показано, что на стадии развитой пластической деформации (РПД) поликристаллов СД ДП инициируют цепочку сложных структурных превращений, в конечном итоге приводящую к явлению фрагментации. Иными словами, - к превращению в ходе РПД первоначально однородно ориентированных зерен в конгломераты, состоящие из множества сплошносопряженных средне и сильно разориентированных микрообластей (фрагментов), средний поперечный размер которых обычно составляет 0.2-0.3 $\mu \mathrm{m}$ [4-8].

Появление СД ДП в пластически деформируемых поликристаллах должно сказываться и на особенностях их деформационного упрочнения. Однако до сих пор этот важнейший аспект физики РПД оставался неизученным. В настоящей работе мы впервые рассмотрим его и в рамках простейшей 2D-модели оценим вклад СД ДП в деформирующее напряжение, а также проанализируем особенности формирования в упругом поле стыковой дисклинации плоского дислокационного скопления и условия прохождения пластического сдвига мимо СД ДП [2,9].

\section{1. Одиночная дислокация}

Рассмотрим трикристалл, находящийся в однородном внешнем поле упругих напряжений $\sigma^{\mathrm{ext}}$ и содержащий клиновую стыковую дисклинацию мощностью $\omega$. Поместим в точке, обозначаемой радиусом-вектором $\mathbf{R}_{p}$, пробную краевую дислокацию (рис. 1) и проанализируем силу $F^{\text {tot }}\left(\mathbf{R}_{p}\right)$, действующую на нее в системе скольжения (n, b):

$$
F^{\mathrm{tot}}\left(\mathbf{R}_{p}\right)=\mathbf{n}\left[\sigma^{\mathrm{ext}}+\sigma^{\mathrm{disl}}\left(\mathbf{R}_{p}\right)\right] \cdot \mathbf{b},
$$

где $\sigma^{\text {disl }}\left(\mathbf{R}_{p}\right)$ - тензор-функция упругих напряжений, генерируемых стыковой дисклинацией в точке нахождения пробной дислокации. Для геометрии, изображенной на рис. 1, представляют интерес лишь компоненты $(x y)$ соответствующих напряжений. Представленные в инвариантной форме, а также в декартовой и полярной системах координат эти компоненты равны

$$
\sigma_{x y}^{\mathrm{ext}} \equiv \frac{1}{b}\left(\mathbf{n} \cdot \sigma^{\mathrm{ext}} \cdot \mathbf{b}\right)=b \sigma_{x y}^{\mathrm{ext}}
$$

$\sigma_{x y}^{\mathrm{disl}} \equiv \frac{1}{b}\left(\mathbf{n} \cdot \sigma^{\mathrm{discl}} \cdot \mathbf{b}\right)=-D \omega \frac{x y}{\left(x^{2}+y^{2}\right)}=-\frac{D \omega}{2} \sin (2 \varphi)$,

где $D=\frac{G}{2 \pi(1-v)}, G-$ модуль сдвига, $v-$ коэффициент Пуассона, $\varphi$ - угол в полярной системе координат.

Сила $b \sigma_{x y}^{\text {ext }}$, действующая на дислокацию со стороны внешнего поля, однонаправлена и не зависит от координат пробной точки. В то же время сила $b \sigma_{x y}^{\text {discl }}$, действующая на нее со стороны упругого поля СД ДП, зависит от местоположения пробной дислокации, причем она положительна слева от дисклинации $(x<0)$ и отрицательна справа от нее $(x>0)$. При $x<0$ обе силы действуют однонаправленно, а при $x>0-$ в противоположные стороны. В области $x<0$ решеточная дислокация ускоряется упругим полем СД ДП, а в области $x>0$ тормозится им. При этом максимальную силу сопротивления дислокация испытывает на луче, проведенном через СД ДП под углом $\varphi=45^{\circ}$. По этой причине краевая решеточная дислокация может пересечь кристалл, содержащий СД ДП только в том случае, если выполняется условие

$$
\sigma_{x y}^{\mathrm{ext}}>\tau_{0}+0.5 D \omega .
$$




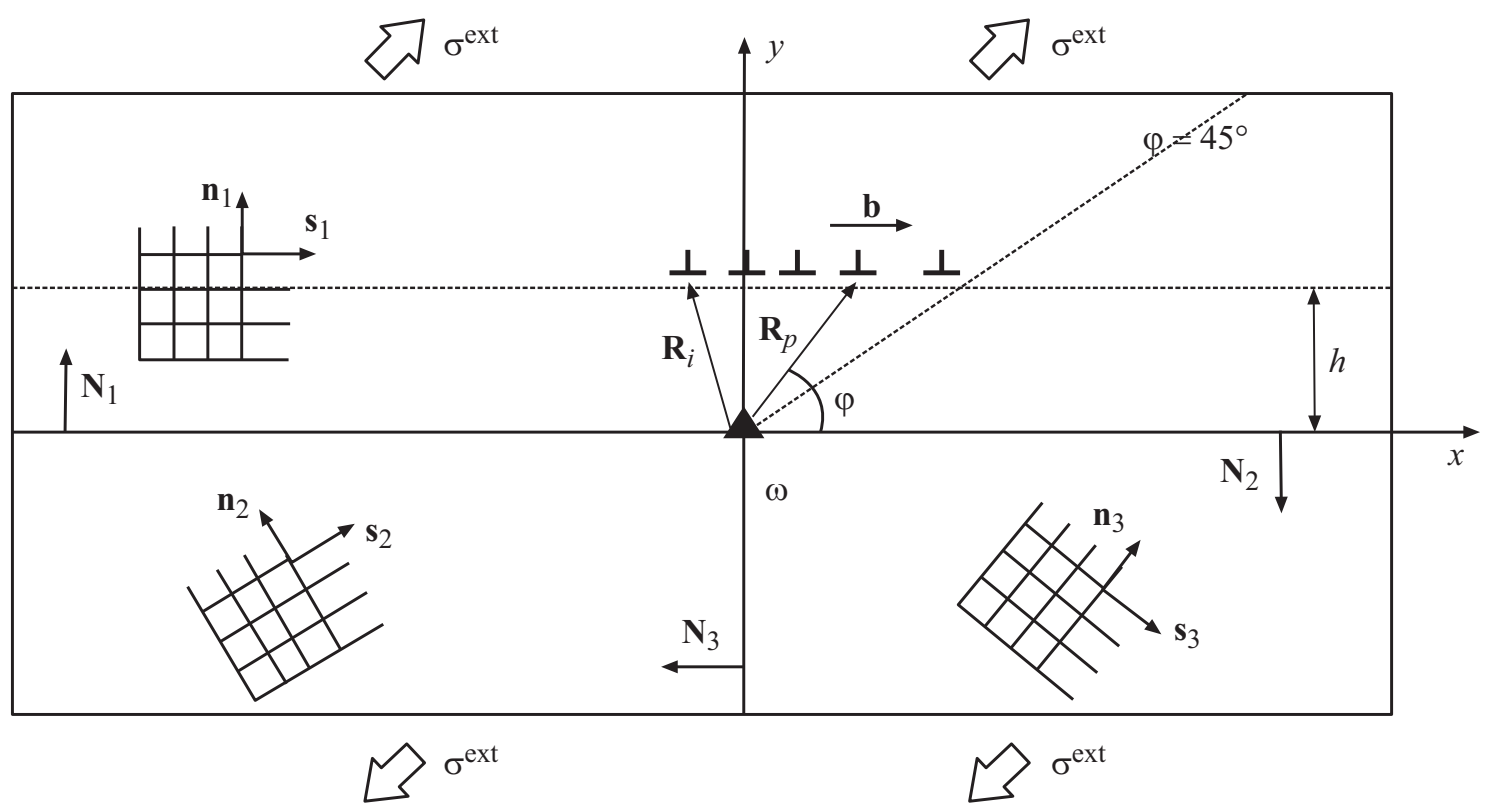

Рис. 1. Схематическое представление модельного трикристалла и геометрии решеточного скольжения.

Слагаемое $\tau_{0}$ в формуле (3) описывает торможение прямолинейной дислокации при ее скольжении по кристаллической решетке зерна в отсутствие СД ДП. Величину его определяют силы Пайерлса, твердорастворное упрочнение, а также взаимодействие с окружающими дислокациями [10]. Учет этих вкладов дает для $\tau_{0}$ величину порядка $10^{-4} \mathrm{G}$, что хорошо согласуется с экспериментально измеряемыми значениями деформирующего напряжения технически чистой меди на начальных стадиях ее пластического течения (т.е. при $\varepsilon \approx 0.01-0.02$ ) [11], но существенно меньше значений, типичных для стадии РПД. При $\varepsilon \geq 0.2$ деформирующее напряжение меди находится на уровне $\left(10^{-3}-10^{-2}\right) G[4]$. В рамках классической дислокационной физики прочности столь резкое повышение деформирующего напряжения связывают с соответствующим повышением при росте $\varepsilon$ плотности однородно ${ }^{1}$ распределенных по объему кристалла решеточных дислокаций [12]. Проведенный выше анализ показывает, однако, что такое же приращение деформирующего напряжения может обеспечить и другой, ранее неучитываемый фактор - появление в структуре пластически деформируемых поликристаллов стыковых дисклинаций деформационного происхождения. Эти линейные мезодефекты ротационного типа формируются на начальных этапах совместной ПД поликристаллического агрегата и монотонно наращивают свою мощность $\omega$ по мере дальнейшего увеличения $\varepsilon$. Эксперименты [13] и теоретические оценки [14] показывают, что при $\varepsilon \geq 0.2$, т. е. на начальной стадии РПД, мощность стыковых дисклинаций $\omega$ составляет примерно $0.2-0.3 \mathrm{rad}$. Подставляя эти значе-

\footnotetext{
${ }^{1}$ Или в составе дислокационных границ слаборазориентированной ячеистой структуры
}

ния в (3), получаем оценку деформирующего напряжения для стадии РПД: $\sigma_{x y}^{\text {ext }}>\tau_{0}+0.5 D \omega \approx 0.25 \mathrm{G} \cdot 10^{-2}$.

\section{2. Плоское дислокационное скопление}

Если условие (3) не выполняется, пробная дислокация останавливается упругим полем СД ДП. При этом, однако, работа источника дислокаций, расположенного на границе кристалла, может продолжаться. В этом случае внутри кристалла начнет формироваться плоское дислокационное скопление. Сила, действующая на пробную дислокацию, будет нарастать и, в конечном итоге, когда число дислокаций в скоплении $N$ достигнет критической величины $N_{c}$, позволит ей преодолеть тормозящее влияние поля стыковой дисклинации. Воспользовавшись ранее разработанными методами 2D-компьютерного моделирования ансамблей взаимодействующих дислокаций в упругом поле СД ДП [15-18], проанализируем детали этого процесса.

Рассмотрим скопление из $N$ дислокаций с вектором Бюргерса $\mathbf{b}=b \cdot \mathbf{s}_{1} \quad\left(\mathbf{s}_{1}-\right.$ единичный вектор), расположенных в зерне 1 в точках с координатами $\mathbf{R}_{i}$ $(i=1,2, \ldots, N)$ на плоскости скольжения $\mathbf{n}_{1}$, отстоящей от СД ДП на расстояние $h$ (рис. 1). На каждую такую дислокацию помимо сил (1), действующих со стороны внешних напряжений и собственных напряжений СД ДП, действует еще и конфигурационная сила $F^{\text {conf }}$, описывающая ее взаимодействие с другими $(N-1)$ дислокациями скопления. Конфигурационная сила, действующая на пробную дислокацию в плоскости 
$a$
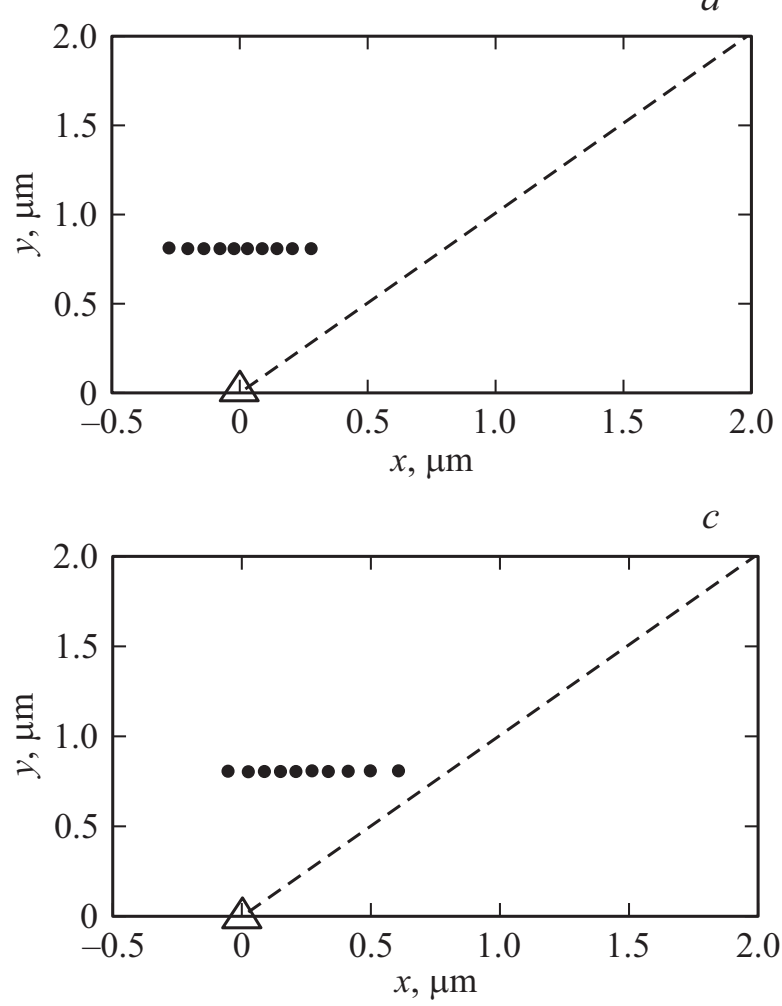
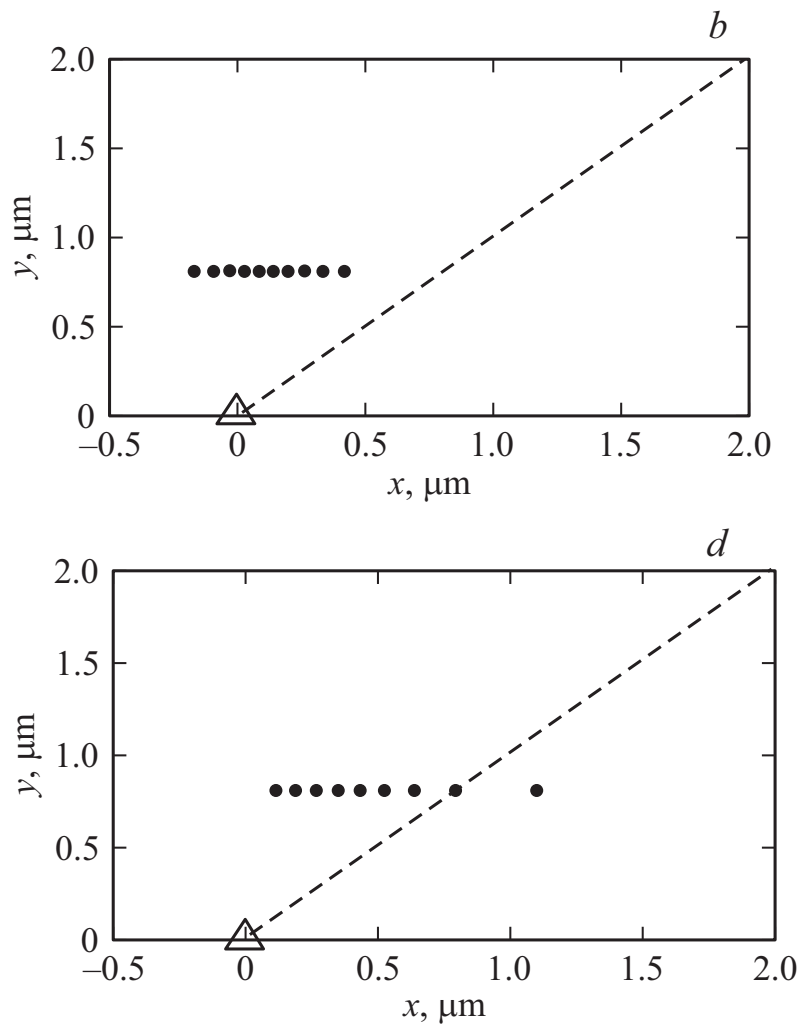

Рис. 2. Равновесная конфигурация скопления в суммарном поле внешних напряжений $\sigma^{\text {ехт }}$ и поле дисклинации мощности $\omega=0.04: a-\sigma^{\mathrm{ext}}=0 ; b-\sigma^{\mathrm{ext}}=0.005 D, c-\sigma^{\mathrm{ext}}=0.01 D$ и $d-$ равновесное скопление после ухода головной дислокации при $\sigma^{\text {ext }}=0.015 D$. Штриховой линией обозначен луч $\varphi=45^{\circ}$ (в полярных координатах), на котором тормозящее напряжение от дисклинации максимально и равно $D \omega / 2$.

ее скольжения, равна

$$
F^{\mathrm{conf}}\left(\mathbf{R}_{p}\right)=\mathbf{n}_{1} \cdot \sum_{i \neq p}^{N} \mathbf{G}\left(\mathbf{R}_{p}, \mathbf{R}_{i}\right) \cdot \mathbf{b},
$$

где $\mathbf{G}\left(\mathbf{R}_{p}, \mathbf{R}_{i}\right)$ - тензор-функция упругих напряжений, создаваемых в точке $\mathbf{R}_{p}$ краевой дислокацией, расположенной в точке $\mathbf{R}_{i}$. Таким образом, результирующая сила, действующая на пробную дислокацию скопления, равна

$$
F_{\text {pup }}^{\mathrm{tot}}\left(\mathbf{R}_{p}\right)=F^{\mathrm{tot}}\left(\mathbf{R}_{p}\right)+F^{\mathrm{conf}}\left(\mathbf{R}_{p}\right) .
$$

Зная результирующую силу, можно вычислить скорость скольжения $V\left(\mathbf{R}_{p}\right)$ пробной дислокации. В квазивязком приближении она равна

$$
V\left(\mathbf{R}_{p}\right)=M \cdot F_{\text {pup }}^{\mathrm{tot}}\left(\mathbf{R}_{p}\right),
$$

где $M$ - коэффициент подвижности дислокации.

\section{3. Результаты компьютерного моделирования плоского дислокационного скопления в упругом поле СД ДП}

Процесс формирования плоского дислокационного скопления в упругом поле СД ДП моделировали посред- ством последовательного испускания положительных дислокаций источником, расположенным на левой боковой границе пробного кристалла. Для избежания динамических эффектов $[19,20]$ каждую следующую дислокацию испускали только после установления равновесия в скоплении, состоящем из ранее испущенных дислокаций. Расчет равновесной конфигурации проводили методом последовательных итераций, разбивая время перехода к равновесию на отрезки, обеспечивающие оптимально малые перемещения дислокаций при заданной их подвижности $M$. Равновесной считалась конфигурация, при которой силы, действующие на каждую из дислокаций скопления, обращались в ноль. Критерием потери устойчивости скопления и прохождения пластического сдвига через силовой барьер, создаваемый СД ДП, являлся „отрыв“ головной дислокации от скопления и последующий выход ее на правую боковую поверхность пробного кристалла.

\section{1. Влияние $\sigma^{\text {ext }}$ на равновесную конфигурацию скопления}

Результаты расчетов равновесной конфигурации скопления, состоящего из десяти дислокаций, для плоскости скольжения, расположенной на расстоянии $h=0.8 \mu \mathrm{m}$ 


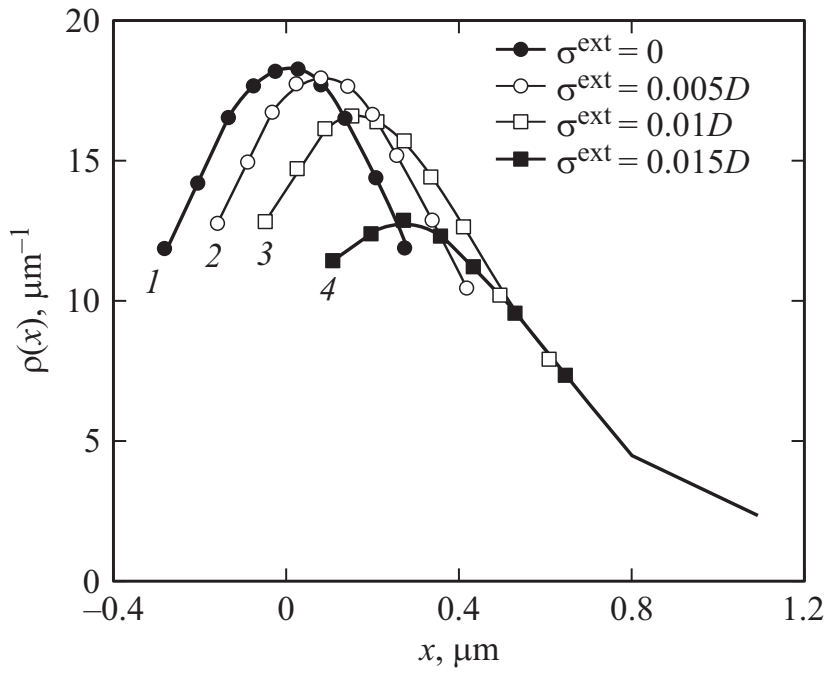

Рис. 3. Распределение плотности дислокаций в скоплении при различных значениях внешнего напряжения $\sigma^{\mathrm{ext}}$ (при $\omega=0.04, h=0.8 \mu \mathrm{m})$. Кривые $1-3$ соответствуют скоплению из 10 дислокаций, кривая 4 - скоплению из 9 дислокаций, остающихся после потери устойчивости и ухода головной дислокации $\left(\sigma^{\mathrm{ext}}>\sigma_{c}\right)$.

от стыковой дисклинации с мощностью $\omega=0.04$, а также зависимости распределения равновесной линейной плотности дислокаций в скоплении $\rho\left(x_{i}\right)$ при различных значениях внешнего напряжения $\sigma^{\text {ext }}$ приведены на рис. 2 и 3 соответственно. Линейную плотность дислокаций в точке $x_{i}$ аппроксимировали формулой

$$
\rho\left(x_{i}\right)=\frac{2}{x_{i+1}-x_{i-1}}, \quad i=2,3, \ldots N-1,
$$

где $x_{i}$ - известные из численного расчета координаты дислокаций равновесного скопления. Значения $\rho\left(x_{i}\right)$ и $\rho\left(x_{N}\right)$ определяли из условия равенства площади под кривой $\rho(x)$ в интервале $\left[x_{1}, x_{N}\right]$ числу дислокаций в скоплении.

Осевая симметрия поля упругих напряжений дисклинации приводит к тому, что в отсутствие внешних напряжений $\left(\sigma^{\mathrm{ext}}=0\right)$ плоское скопление дислокаций располагается симметрично относительно оси $O Y$ декартовой системы координат (рис. 2, $a$ ). При увеличении внешнего напряжения $\sigma^{\text {ext }}$ дислокационное скопление смещается как целое и его форма становится все более асимметричной (рис. 2, $b, c$; кривые 2 и 3 на рис. 3). Наконец, при напряжении $\sigma^{\mathrm{ext}}$, большем критического $\sigma_{c}=0.0145 D$, происходит потеря устойчивости скопления, его головная дислокация „отрывается“ от скопления и уходит из кристалла. При этом происходит релаксационная перестройка оставшихся дислокаций скопления в новую равновесную конфигурацию (рис. $3, d$, кривая 4 на рис. 2). Добавление к ней следующей дислокации при срабатывании источника приводит к тому, что головная дислокация скопления вновь потеряет устойчивость и уйдет на сток.

\section{2. Влияние $\boldsymbol{N}$ и $\boldsymbol{h}$ на величину критического напряжения сдвига $\sigma_{c}$}

Результаты расчетов зависимости $\sigma_{c}$ от $h$ представлены на рис. 4. При фиксированном числе дислокаций $(N)$ в скоплении величина $\sigma_{c}$ монотонно нарастает по мере удаления плоскости скольжения от дисклинации, а при больших $h$ зависимость $\sigma_{c}(h)$ асимптотически стремится к насыщению. При заданном $h$ величина $\sigma_{c}$ увеличивается с уменьшением числа дислокаций в скоплении и достигает максимального значения $\sigma_{c}^{*}=D \omega / 2$ при $N=1$. Наконец, при заданных $\sigma^{\mathrm{ext}}$ и $h$ прохождение пластического сдвига через силовой барьер, создаваемый дисклинацией, может осуществляться лишь при числе дислокаций в скоплении, большем некоторого критического $N_{c}$. Результаты расчетов зависимости $N_{c}(h)$ при различных величинах $\sigma^{\text {ext }}$ приведены на рис. 5. Видно, что эта зависимость близка к линейной и асимптоти-

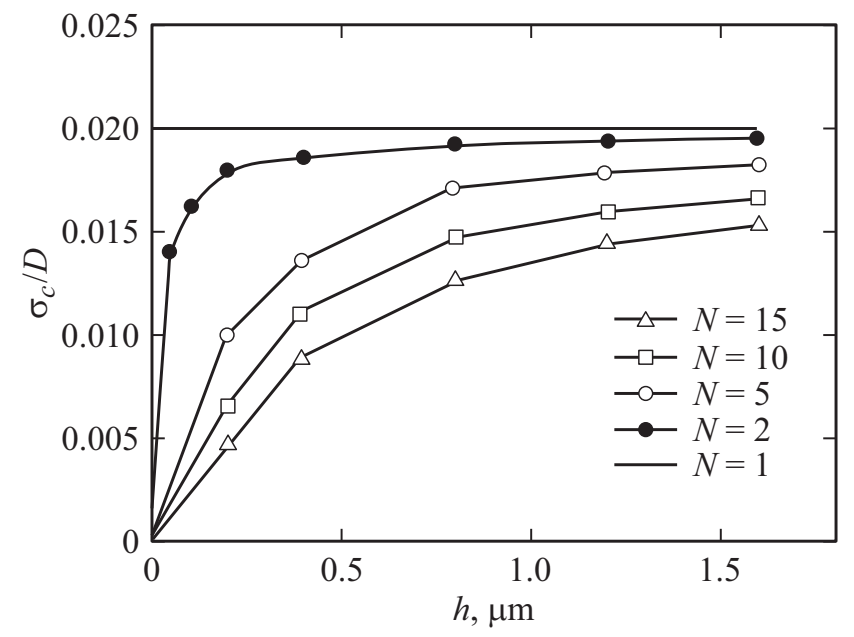

Рис. 4. Зависимости критического напряжения $\sigma_{c}$, при котором происходит потеря устойчивости скопления, от расстояния $h$ до стыковой дисклинации мощности $\omega=0.04$.

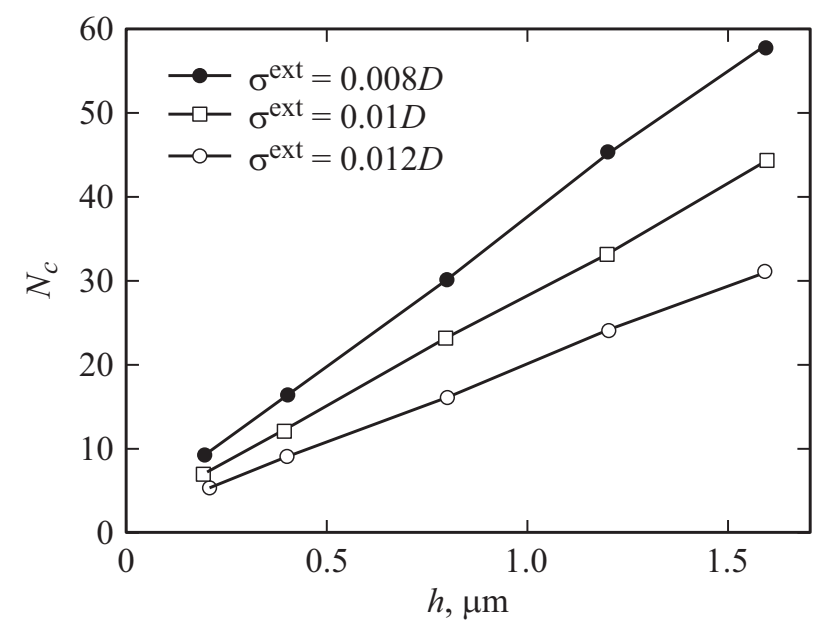

Рис. 5. Зависимость максимального количества дислокаций $N$ в равновесном скоплении от расстояния $h$ между плоскостью скольжения и стыковой дисклинацией мощности $\omega=0.04$ при различных значениях внешнего напряжения. 
чески стремится к единице при $\sigma^{\mathrm{ext}} \rightarrow D \omega / 2$. Величина $N_{c}$ является, очевидно, максимально возможной при заданных $\sigma^{\text {ext }}$ и $h$ числом дислокаций в скоплении, при котором еще возможно его равновесие.

\section{Заключение}

Из проведенного анализа следует, что в процессе пластической деформации поликристалла в теле зерен возможно образование плоских скоплений дислокаций в отсутствие каких-либо видимых физических барьеров, расположенных в плоскости скольжения дислокаций. Появление таких скоплений может быть обусловлено возникновением в ходе пластической деформации в тройных стыках и вершинах плоских фасеток границ зерен стыковых дисклинаций деформационного происхождения, создающих силовой барьер для прохождения дислокаций. Одной из отличительных черт таких скоплений является то, что они не исчезают при снятии внешнего напряжения. Другим отличительным признаком является характерная колоколообразная форма распределения в них плотности дислокаций: максимальное сгущение происходит не на краю, как в классических заторможенных вблизи барьеров плоских дислокационных скоплениях [10], а в их центральной части.

В заключение следует отметить еще одну особенность торможения дислокаций в поле стыковой дисклинации. Критическое напряжение $\sigma_{c}$ прохождения мимо нее одиночной дислокации не зависит от того, на каком расстоянии $h$ от стыковой дисклинации находится плоскость ее скольжения. Напротив, в случае плоского скопления нескольких таких же дислокаций оно оказывается тем меньше, чем меньше $h$. При увеличении $h$ критическое напряжение $\sigma_{c}$ возрастает, стремясь к насыщению. Таким образом, при локализации пластической деформации наибольший упрочняющий эффект от упругих полей дисклинации достигается не вблизи стыков и изломов границ, а вдали от них.

Исследование выполнено за счет гранта Российского научного фонда (проект № 15-13-20030).

\section{Список литературы}

[1] Рыбин В.В., Зисман А.А., Золоторевский Н.Ю. // ФТТ. 1985. Т. 27. Вып. 1. С. $181-186$.

[2] Рыбин В.В., Перевезенцев В.Н., Свирина Ю.В. // ЖТФ. Т. 86. Вып. 6. С. 100-105.

(Rybin V.V., Perevezentsev V.N., Svirina J.V. // Technical Physics. 2016. Vol. 61. N 6. P. 898-903.

[3] Romanov A.E., Vladimirov V.I. Disclinations in Crystalline Solids. in F.R.N. Nabarro ed. „Dislocations in solids“, NorthHolland, Amsterdam, 1992. Vol. 9. P. 191-402.

[4] Рыбин В.В. Большие пластические деформации и разрушение металлов. М.: Металлургия, 1986. 224 с.

[5] Rybin V.V. // Problems of Material Science. 2003. Vol. 33. N 1. P. $9-28$.
[6] Сарафанов Г.Ф., Перевезенцев В.Н., Рыбин В.В. Основы кинетической теории формирования разориентированных структур при пластической деформации металлов. Н. Новгород: Литера, 2011. 368 с.

[7] Romanov A.E., Kolesnikova A.L. // Progress in Mater. Sci. 2009. Vol. 54. P. 740-769.

[8] Estrin Y., Vinogradov A. // Acta Mater. 2013. Vol. 61. P. 782-817.

[9] Рыбин В.В., Перевезенцев В.Н., Свирина Ю.В. // ЖТФ. 2017. Т. 87. Вып. 5. Р. 726-735.

[10] Хирт Дж., Лоте И. Теория дислокаций. М.: Атомиздат, $1972.599 \mathrm{c}$.

[11] Физическое металловедение. Т. 3: Физико-механические свойства металлов и сплавов / Под ред. Р.У. Кана, П. Хаазена. М.: Металлургия, 1987. 661 с.

[12] Штремель М.А. Прочность сплавов. Ч. 1. Дефекты решетки. М.: Металлургия, 1982. 280 с.

[13] Klemm V., Klimanek P., Seeafeldt M. // Phys. Stat. Sol. (A). 1999. Vol. 175. P. 569-576.

[14] Rybin V.V., Zisman A.A., Zolotorevsky N.Yu. // Acta Metall. Mater. 1993. Vol. 41. N 7. P. 2211-2217.

[15] Van der Giessen E., Needleman A. // Model Simul. Mater. Sci. Eng. 1995. Vol. 3. P. 689-735.

[16] Aubry S., Rhee M., Hommes G., Bulatov V.V., Arsenlis A. // J. Mech. Phys. Sol. Vol. 94. 2016. P. 105-126.

[17] Kubin L., Devincre B., Hoc T. //Acta Mater. 2008. Vol. 56. N 20. P. 6040-6049.

[18] Arsenlis A., Cai W., Tang M., Rhee M., Oppelstrup T., Hommes G., Pierce1 T.G., Bulatov V.V. // Modelling Simul. Mater. Sci. Eng. 2007. Vol. 15. P. 553-595.

[19] Gurrutxaga-Lerma B., Balint D.S., Dini D., Eakins D.E., Sutton A.P. // Proc. Roy. Soc. A. 2013. Vol. 469. P. 20130141.

[20] Rzhavtsev E.A., Gutkin M.Yu. // Scripta Mater. 2015. Vol. 100. P. 102-105. 